\title{
Atuação do enfermeiro na assistência ao paciente idoso portador de úlcera venosa
}

The nurse's performance in the attendance to the patient senior bearer of veined ulcer

Papel de la enfermera en el portador de la asistencia al paciente anciano de úlcera venosa

Aline Pereira Bonfim ${ }^{1 *}$, Gilvane Teixeira Souzaํ, Márcia Carvalho Pita ${ }^{1}$, Anne Jacob de Souza Araújo².

\section{RESUMO}

Objetivo: Descrever o perfil clínico dos pacientes idosos portadores de úlcera venosa e a assistência que vem sendo prestada pelos enfermeiros nas unidades de saúde. Métodos: Trata-se de uma revisão bibliográfica, que ocorreu na base de dados Biblioteca Virtual em Saúde, utilizando-se os descritores em saúde "idoso", "úlcera varicosa", "cuidados de enfermagem", combinados entre si. Foram selecionados 12 artigos que se fizeram apropriados a esta pesquisa. Resultados: Conhecer o perfil sociodemográfico dos pacientes e sua relação com a qualidade dos atendimentos prestados pelos enfermeiros viabiliza um bom prognóstico no tratamento dessas úlceras. Considerações finais: Percebeu-se que a atividade continuada e a implantação de protocolos são a melhor estratégia para um bom atendimento evitando assim recidivas.

Palavras-chave: Idoso, Úlcera varicosa, Cuidados de enfermagem.

\begin{abstract}
Objective: To describe the clinical profile of elderly patients with venous ulcer and the care provided by nurses in health units. Methods: This is a bibliographical review, which was carried out in the Virtual Health Library database, using the descriptors in "elderly", "varicose ulcer" and "nursing care", combined with each other. We selected 12 articles that were appropriate for this research. Results: Knowing the sociodemographic profile of the patients and their relationship with the quality of care provided by the nurses allows a good prognosis in the treatment of these ulcers. Final considerations: It was noticed that the continuous activity and the implantation of protocols is the best strategy for a good care, thus avoiding relapses.
\end{abstract}

Keywords: Elderly, Varicose ulcer, Nursing care.

\section{RESUMEN}

Objetivo: Describir el perfil clínico de los pacientes ancianos portadores de úlcera venosa y la asistencia que vienen siendo prestada por los enfermeros en las unidades de salud. Métodos: Se trata de una revisión bibliográfica, que ocurrió en la base de datos Biblioteca Virtual en Salud, utilizando los descriptores en salud "anciano", "úlcera varicosa", "cuidados de enfermería", combinados entre sí. Se seleccionaron 12 artículos que se hicieron apropiados a esta investigación. Resultados: Conocer el perfil sociodemográfico de los pacientes y su relación con la calidad de las atenciones prestadas por los enfermeros viabiliza un buen pronóstico en el tratamiento de esas úlceras. Consideraciones finales: Se percibió que la actividad continuada y la implantación de protocolos es la mejor estrategia para una buena atención evitando así recidivas.

Palabras clave: Ancianos, Úlcera varicosa, Cuidados de enfermería.

\footnotetext{
${ }^{1}$ Enfermeira, Centro Universitário Jorge Amado, Salvador, Bahia. *E-mail: Ilynny20@hotmail.com

${ }^{2}$ Discente do Centro Universitário Jorge Amado, Salvador, Bahia.
} 


\section{INTRODUÇÃO}

O aumento do número de idosos é notável em todo o mundo. Em 2010, existiam no país 20,5 milhões de idosos, aproximadamente 39 para cada grupo de 100 jovens. Estimam-se para 2040, mais que o dobro, representando $23,8 \%$ da população brasileira e uma proporção de quase 153 idosos para cada 100 jovens. Essa nova realidade demográfica, com um número cada vez maior de idosos, exige também do sistema de saúde capacidade para responder às demandas atuais e futuras. Com avanço da tecnologia junto com uma melhor qualidade de vida, possibilitou ao homem uma expectativa de vida maior, consequentemente, o aumento da preocupação não só com a saúde física, mas também com a saúde mental da população com mais de 65 anos de idade. A partir dessa idade surgem doenças típicas da população idosa somadas à comorbidades pré-existentes (SANTANA RF, et al., 2009; MIRANDA GMD, et al., 2016).

Segundo o Ministério da Saúde (2008), as doenças associadas à velhice não fazem parte do processo normal de envelhecimento. Elas ocorrem porque as perdas funcionais decorrentes do envelhecimento aumentam a vulnerabilidade do idoso ao câncer, às doenças cardíacas, ao acidente vascular encefálico e outras. E neste contexto, uma das principais causas que levam idosos à internação hospitalar são as doenças do aparelho circulatório, perfazendo um total de $27,4 \%$ das internações.

As úlceras são mais frequentemente encontradas nos serviços da rede básica de saúde, hospitais gerais e especializados e advém da insuficiência venosa crônica (IVC), em um percentual entre $80 \%$ a $85 \%$, e de doença arterial $5 \%$ a $10 \%$, sendo o restante de origem neuropática (usualmente diabética) ou mista (ABBADE LPF e LASTORIA S, 2006).

Estima-se que aproximadamente $3 \%$ da população brasileira possuam lesões venosas, que se elevam para $10 \%$ no caso de diabéticos. Dessa população, em torno de quatro milhões de pessoas possuem lesões crônicas ou têm algum tipo de complicação no processo de cicatrização (FERNANDES LF, et al., 2013).

As principais causas das úlceras crônicas no idoso são de origem vascular, resultantes da insuficiência venosa crônica (70 a 90\%) e com uma menor frequência de origem neuropática e arterial (10 a 15\%). Afetam de forma significativa, a qualidade de vida dos seus portadores, pois além do seu caráter redicivante, permanecem por muito tempo abertas, influenciando nas relações sociais, no exercício do trabalho e nas atividades de lazer (SILVA FAA, et al., 2009).

As pessoas com lesões de qualquer etiologia requerem uma assistência de qualidade com visão integral do ser humano, dentro do seu contexto socioeconômico, cultural e de saúde, e com atuação de equipe multidisciplinar, considerando a complexidade e dinamicidade que envolve o processo de cicatrização dessas lesões (ABBADE LPF e LASTORIA S, 2006).

O conhecimento dessas doenças é extremamente importante para o diagnóstico correto para que medidas terapêuticas específicas sejam adotadas. O diagnóstico incorreto desencadeará necessariamente uma abordagem incorreta, com atraso na cicatrização da úlcera e prejuízos ao paciente. Por exemplo, se um paciente com úlcera arterial receber compressão elástica, pois foi erroneamente diagnosticado como úlcera venosa, poderá ocorrer uma descompensação importante da circulação arterial periférica, causando piora da dor, aumento e surgimento de novas úlceras e formação de áreas necróticas, levando muitas vezes à amputação do membro comprometido (MALAGUTTI W e KAKIHARA TC, 2011).

Este trabalho tem como justificativa a importância da assistência sistemática com o público idoso com úlcera venosa, afim de diminuir o tempo de tratamento, evitar recidivas, e consequentente, complicações relacionadas a essas lesões. Busca-se o aprimoramento das técnicas já utilizadas, afim de sistematizar 0 tratamento.

Diante desse contexto e procurando entender sobre a real situação o estudo tem como questão norteadora: Padronizar as condutas que deverão ser seguidas pelos enfermeiros nos cuidados com o paciente idoso portador de úlcera venosa poderá melhorar a qualidade do atendimento? 
O estudo tem como objetivos identificar o perfil clínico dos pacientes idosos portadores de úlcera venosa e conhecer a forma que a assistência vem sendo prestada pelos enfermeiros nas unidades de saúde aos idosos portadores de úlcera venosa.

\section{METODOLOGIA}

Foi realizada uma revisão integrativa de literatura. Teve por finalidade juntar, organizar e avaliar de forma sistemática resultados de pesquisas voltados para uma determinada temática que permitirá a síntese do conhecimento (OLIVEIRA FP, et al., 2016).

As buscas foram realizadas nos seguintes bancos de dados Biblioteca Virtual em Saúde (BVS). A coleta foi realizada utilizando a associação dos seguintes descritores em Ciências da Saúde (Decs): idoso, úlcera varicosa e cuidados de enfermagem, no período de fevereiro de 2018.

Dos descritores associados resultaram em um total de 40 artigos. Foram selecionados os que atendiam aos seguintes critérios de inclusão: artigos com textos completos, em português, entre 2006 e 2016, e descartados os que atendiam ao seguinte critério de exclusão: artigos que não abordassem a temática do estudo como, artigos de revisão, teses e dissertações.

Após passar pelos critérios de inclusão e exclusão restaram 34 artigos aos quais foram lidos os títulos e selecionados os que condiziam com o tema do trabalho, restando 21 artigos.

Após essa seleção foram lidos os resumos de forma minuciosa para identificar os que atendiam ao objetivo da pesquisa, de forma que restaram 17 artigos. Foram excluídos repetidos e incompletos 05 , restando assim 12 artigos que se fizeram apropriados a esta pesquisa.

Foi realizada a análise do conteúdo, a partir da realização de fichamentos e estruturação de categorias.

O presente estudo por ser de revisão não foi submetido à avaliação do Comitê de Ética em Pesquisa de acordo com a Resolução 466/12 do Conselho Nacional de Saúde (CNS), por se tratar de uma revisão da literatura, porém todos os preceitos éticos estabelecidos serão respeitados no que se refere a zelar pela legitimidade das informações de acordo com as normas da Associação Brasileira de Normas Técnicas (ABNT).

\section{RESULTADOS E DISCUSSÃO}

Nesta revisão bibliográfica foram analisados 12 trabalhos relacionados ao objetivo da pesquisa. Para facilitar a apresentação e análise destes resultados, elaborou-se uma tabela com os seguintes dados: autores/as, ano de publicação, título, objetivo da pesquisa (Tabela 1).

A abordagem dos assuntos discutidos nos artigos utilizados, envolvem o perfil sociodemográfico dos pacientes idosos com úlcera venosa e sua relação com a qualidade dos atendimentos prestados pelos enfermeiros, visando a importância de se estabelecer um protocolo de assistência eficaz e da educação continuada e permanente desses profissionais no exercício das suas funções.

Diante da leitura do material descrito na tabela acima, foi formulada as seguintes categorias: perfil clínico dos pacientes idosos portadores de úlcera venosa, e características do atendimento realizado pelo enfermeiro nas unidades de saúde visando melhoria no atendimento do idoso portador de úlcera venosa.

\section{Perfil clínico dos pacientes idosos portadores de úlcera venosa}

Benevides JP, et al. (2013), identificou que a maioria dos idosos que possuem úlcera venosa ou arterial encontram-se numa faixa etária de 69 anos, a maior parte deste são do sexo feminino e casados. Em relação ao grau de escolaridade, nunca tinham estudado, ou apenas estudaram cerca de cinco anos em toda a vida. 
Tabela 1 - Artigos originais selecionados.

\begin{tabular}{|c|c|c|c|}
\hline Autores/as & Ano & Título & Objetivo(s) \\
\hline Benevides JP, et al. & 2012 & $\begin{array}{l}\text { Avaliação clínica de úlcera de perna em } \\
\text { idosos. }\end{array}$ & $\begin{array}{l}\text { Realizar avaliação clínica de úlceras } \\
\text { de perna em idosos atendidos em um } \\
\text { ambulatório especializado. }\end{array}$ \\
\hline Silva $\mathrm{MH}$, et al. & 2013 & $\begin{array}{l}\text { O cotidiano do homem que convive com } \\
\text { a úlcera venosa crônica: estudo fenome- } \\
\text { nológico. }\end{array}$ & $\begin{array}{l}\text { Compreender o cotidiano do homem } \\
\text { que convive com a úlcera venosa crô- } \\
\text { nica. }\end{array}$ \\
\hline Dantas DV, et al. & 2013 & $\begin{array}{l}\text { Proposta de protocolo para assistência } \\
\text { as pessoas com úlcera venosa. }\end{array}$ & $\begin{array}{l}\text { Propor um protocolo para assistência } \\
\text { às pessoas com úlcera venosa (UV) } \\
\text { atendidas em Hospital de referência } \\
\text { do Rio Grande do Norte/RN, Brasil. }\end{array}$ \\
\hline Reis DB, et al. & 2013 & $\begin{array}{l}\text { Cuidado às pessoas com úlcera venosa: } \\
\text { percepção dos enfermeiros da estratégia } \\
\text { de saúde da família. }\end{array}$ & $\begin{array}{l}\text { Identificar o conhecimento dos enfer- } \\
\text { meiros das equipes de saúde da fa- } \\
\text { mília (ESF) do distrito III de Uberaba- } \\
\text { MG sobre os cuidados necessários } \\
\text { às pessoas portadoras de úlcera ve- } \\
\text { nosas e descrever suas percepções. }\end{array}$ \\
\hline Dantas DV, et al. & 2013 & $\begin{array}{l}\text { Protocolo de assistência a pessoas com } \\
\text { úlcera venosa: validação do conteúdo }\end{array}$ & $\begin{array}{l}\text { Identificar aspectos validados por juí- } \\
\text { zes para elaboração de protocolo de } \\
\text { assistência a pessoas com úlceras } \\
\text { venosas. }\end{array}$ \\
\hline Dias TYAF, et al. & 2013 & $\begin{array}{c}\text { Influência da assistência e características } \\
\text { clínica na qualidade de vida de portado- } \\
\text { res de úlcera venosa }\end{array}$ & $\begin{array}{c}\text { Verificar a influência da assistência e } \\
\text { das características clínicas na quali- } \\
\text { dade de visa de pacientes com úlcera } \\
\text { venosa. }\end{array}$ \\
\hline Torres VG, et al. & 2014 & $\begin{array}{l}\text { Comparação dos domínios da qualidade } \\
\text { de vida de clientes com úlcera venosa. }\end{array}$ & $\begin{array}{c}\text { Comparar os domínios da qualidade } \\
\text { de vida relacionados à saúde (QVRS) } \\
\text { de pessoas com úlcera venosa (UV) } \\
\text { atendidas no Hospital Universitário } \\
\text { Onofre Lopes (HUOL), em Natal/RN, } \\
\text { Brasil, e em quatro unidades de cui- } \\
\text { dados de saúde primários em Évora, } \\
\text { Portugal. }\end{array}$ \\
\hline Salvetti MG, et al. & 2014 & $\begin{array}{l}\text { Prevalência da dor e fatores associados } \\
\text { em pacientes com úlcera venosa }\end{array}$ & $\begin{array}{l}\text { Identificar a prevalência de dor e veri- } \\
\text { ficar os principais fatores associados } \\
\text { à dor em pacientes com úlceras ve- } \\
\text { nosas. }\end{array}$ \\
\hline Eberhardt TD, et al. & 2016 & $\begin{array}{l}\text { Perfil sociodemográfico e clínico de paci- } \\
\text { entes com úlceras venosas acompanha- } \\
\text { dos em ambulatório: estudo transversal } \\
\text { descritivo. }\end{array}$ & $\begin{array}{l}\text { Identificar o perfil sociodemográfico e } \\
\text { clínico dos pacientes com úlceras ve- } \\
\text { nosas, acompanhados no ambulató- } \\
\text { rio de um hospital universitário do sul } \\
\text { do Brasil. }\end{array}$ \\
\hline Medeiros ABA, et al. & 2016 & $\begin{array}{l}\text { Associação dos fatores socioeconômicos } \\
\text { e clínicos e o resultado integridade tissu- } \\
\text { lar em pacientes com úlceras. }\end{array}$ & $\begin{array}{l}\text { Analisar a associação entre os fato- } \\
\text { res socioeconômicos e clínicos e os } \\
\text { indicadores do resultado de enferma- } \\
\text { gem Integridade Tissular em pacien- } \\
\text { tes com úlceras venosas. }\end{array}$ \\
\hline Oliveira FP, et al. & 2016 & $\begin{array}{l}\text { Classificações de intervenções e resulta- } \\
\text { dos de enfermagem em pacientes com } \\
\text { feridas: mapeamento cruzado }\end{array}$ & $\begin{array}{c}\text { Realizar o mapeamento cruzado dos } \\
\text { termos referentes às intervenções e } \\
\text { aos resultados de enfermagem nos } \\
\text { prontuários dos pacientes com feridas } \\
\text { em comparação às classificações de } \\
\text { intervenções e aos resultados de en- } \\
\text { fermagem. }\end{array}$ \\
\hline Rizzattil SJS, et al. & 2016 & $\begin{array}{l}\text { Cuidados em saúde de pessoas com úl- } \\
\text { cera venosa em assistência ambulatorial }\end{array}$ & $\begin{array}{c}\text { Descrever o cuidado em saúde reali- } \\
\text { zado pelas pessoas com úlcera ve- } \\
\text { nosa em assistência ambulatorial em } \\
\text { um hospital público da região sul do } \\
\text { Brasil. }\end{array}$ \\
\hline
\end{tabular}

Fonte: próprios autores, 2018. 
A maioria dos pacientes com úlcera venosa tem no membro inferior edema, veias varicosas, eritema, descamação e prurido, possuem em média, entre um a cinco lesões, e que o tempo de permanência das mesmas era de 3 anos, apresentava dor de intensidade variável (EBERHARDT TD, et al., 2016).

As úlceras venosas são encontradas com maior frequência, em idosos, na região mais distal dos membros inferiores, como por exemplo, o maléolo. As úlceras nessa região estão intrinsecamente ligadas a deficiência do sistema circulatório (BENEVIDES JP, et al.,2013).

Outro aspecto observado foi que a maioria dos pacientes com úlcera venosa relataram ser portador de hipertensão arterial sistêmica, seguida da insuficiência venosa crônica, além de fazerem uso contínuo de medicamentos, possuíam alterações significativas nos índices de massa corporal sendo em média $29,7 \mathrm{~kg} / \mathrm{m}^{2}$, dados analisados no ambulatório (EBERHARDT TD, et al., 2016).

A dor em pacientes com úlcera venosa é uma característica predominante, apresentando-se pior à noite, gerando limitação na mobilidade dos membros inferiores afetados. É evidenciada por muitos pacientes como o maior impacto em sua qualidade de vida (TORRES GV, et al., 2014).

Devido às restrições nas atividades da vida diária, os portadores de úlcera venosa, são expostos a uma séria de conflitos desencadeando, consequentemente, ao mesmo o confinamento e afastamento da sociedade (SILVA MH, et al.,2013).

Estudos comprovam que a qualidade de vida em pacientes portadores de feridas crônicas, afetam seu estilo de vida devido à dor, dificuldade de mobilidade, depressão, perda da autoestima, isolamento social, inabilidade para o trabalho e frequentemente altera a imagem corporal, proporcionando uma diminuição na qualidade de vida. As úlceras crônicas representam a problemática típica das lesões crônicas e possui caráter quase sempre recidivo (CARMO SS, et al.,2007).

Vale ressaltar que idosos possuem úlceras venosas há mais de um ano, que fatores como infecções tamanho, localização, tipo da úlcera, metabolismo e funcionamento do sistema circulatório contribuem significativamente para a cicatrização destas úlceras (BENEVIDES JP, et al.,2013).

O domínio da dor relativamente pode estar associado com fatores sociodemográficos como: gênero, faixa etária, estado civil, escolaridade, profissão e ocupação, renda, doenças crônicas, sono, etilismo e tabagismo. Pacientes com ocupação profissional evidenciam menor intensidade da dor, ou seja, os cidadãos que exercem uma atividade laborativa propendem a enfatizar não mais que a sensação dolorosa (SALVETTI MG, et al.,2014).

As feridas crônicas causam uma diminuição da autoestima nos pacientes, visto que atingem a vida social, psicológica e emocional destes, levando a um isolamento e abandono do tratamento causando recidivas. Mudanças nas relações interpessoais, perda significativa da autonomia, incapacitação para realização de atividades, baixa autoestima, imagem corporal e autoimagem prejudicadas, trazem reflexos no processo de socialização do homem (TORRES GV, et al., 2014; SILVA MH, et al., 2013).

A úlcera venosa causa inúmeras restrições no cotidiano, afetando a qualidade de vida relacionada à saúde, por ser uma doença de classificação crônica, evidencia largamente restrições em uma parte localizada do corpo, que pode se estender às outras áreas do corpo (DIAS TYAF, et al., 2013).

Existem instruções que o correto gerenciamento da dor contribui realmente para uma boa cicatrização das úlceras. O controle da dor, ao portador de uma úlcera venosa, melhora significativamente a qualidade de vida do indivíduo, pois com isso irá gerar melhora no auto estima, fazendo com que eles fiquem menos predispostos a pensar na dor. A incidência de dor na úlcera venosa em pacientes com maior faixa etária, menor poder aquisitivo e grau de instrução é bem maior. Em relação a assistência prestada na úlcera venosa pode-se observar que o tipo prestado influencia diretamente no grau da dor. O grau da dor pode estar relacionado também com práticas viciantes como consumo do álcool e do tabaco. Conforme o tamanho e aspecto da lesão, diminui ou aumenta a intensidade da dor e um impacto nas atividades diárias (SALVETTI MG, et al., 2014). 
Os sinais e sintomas decorrentes da lesão afetam diretamente na vida social gerando isolamento aos homens portadores de úlcera venosa, a dor crônica, o odor fétido, a lesão propriamente dita, gera afastamentos. A vergonha e o preconceito inviabilizam a vida a dois, e essa falta que eles sentem de ter uma companheira, um apoio, interfere diretamente na sua recuperação (SILVA MH et al.,2013).

A saúde mental de um indivíduo portador de úlcera venosa é severamente comprometida, alterando o seu nível de qualidade de vida, limitando-o de apreciar a vida e realizar suas atividades do dia a dia que requer esforço (TORRES GV, et al., 2014).

Além disso, pessoas portadoras de úlceras venosas desenvolvem um custo adicional nas suas despesas mensais, com o tratamento farmacológico e materiais para os curativos. A depender da situação financeira em que o paciente se encontre inserido, a presença da ferida e os cuidados que esta demanda, cause uma desestabilização na parte financeira que termina afetando a qualidade de vida desses indivíduos (DIAS TYAF, et al., 2013).

A procura pela assistência nos serviços de saúde para a prevenção dos agravos ocorre de maneira desproporcional, as mulheres em sua maioria ajudam a compor essa estatística, enquanto que os homens não comparecem, o reflexo pode ser observado nas intervenções tardias e na presença de complicações do quadro clínico (EBERHARDT TD, et al., 2016).

Foi feita uma correlação entre a renda familiar e o indicador necrose ao qual apontou grandes chances de o tratamento ser negligenciado ou ser realizado de forma pouca eficaz nesses pacientes, aumentando o risco de desenvolver tecidos não viáveis, impedindo assim o processo de cicatrização (MEDEIROS ABA, et al., 2016).

Relacionado a forma que as pessoas portadoras de úlceras venosas cuidam das mesmas, ficou evidenciado a necessidade de aproximação entre o popular e o profissional no sentindo de favorecer a troca de conhecimentos para uma maior compreensão relacionadas às alterações que ocorrem na vida das pessoas (RIZZATTI SJS, et al.,2016).

A qualidade de vida do portador de úlcera venosa é drasticamente alterada, principalmente se este for ativo, gerando um aumento do número de atestados, diminuição na produção de trabalho e até mesmo isolamento social, por conta da aparência (TORRES GV, et al., 2014).

O tipo de assistência prestada ao portador de úlcera venosa influencia drasticamente na sua capacidade funcional. Um atendimento adequado e embasado na parte científica juntamente com a prática profissional diminui a incapacidade funcional e um menor impacto da dor na qualidade de vida (DIAS TYAF, et al.,2013).

A avaliação clínica da úlcera de perna deve ser realizada por uma equipe multidisciplinar capacitada acerca do assunto. A equipe de enfermagem é responsável pelos curativos tanto nas unidades básicas quanto no âmbito hospitalar (BENEVIDES JP, et al.,2013).

O tratamento para úlcera venosa é prolongado e requer alto custo, o que gera uma dependência na terapia. Os pacientes se restringem às atividades cotidiana, devido a cronicidade das mesmas, prolongado o tempo de cura dessas úlceras (TORRES GV, et al., 2014).

O tipo de assistência está correlacionado com as características da lesão o que interfere na capacidade funcional do paciente. Diante desse contexto espera-se que intervenções sejam criadas para melhorar a qualidade de vida dos mesmos (DIAS TYAF, et al.,2013).

Visando um estilo de vida baseado nos bons hábitos de saúde, as pessoas com úlcera venosa mudam seus hábitos alimentares, controlando o sal, açúcar, temperos, frituras, gorduras e carboidratos, assim como ingerindo diariamente frutas, verduras, e cuidando da higiene dos alimentos e pessoal (RIZZATTI SJS, et al.,2016).

Diante da diversidade acerca do perfil dos pacientes idosos com úlceras venosas fica evidenciado que estes não têm conhecimento sobre o problema de saúde que os acomete. Devido à falta de informação e 
orientação os mesmos têm sua rotina diária alterada em vista da doença. Fica evidenciado que a falta de conhecimento sobre a patologia pode gerar diversos agravos à vida social desses pacientes.

\section{Características do atendimento realizado pelo enfermeiro nas unidades de saúde visando melhoria no atendimento do idoso portador de úlcera venosa}

A equipe de enfermagem é responsável pelo cuidado de feridas, seja nas unidades básicas de saúde como em âmbito hospitalar. O enfermeiro se destaca na avaliação clínica e na decisão terapêutica adequada, papel de grande relevância no processo de cicatrização das mesmas (BENEVIDES JP, et al.,2013).

Os enfermeiros surgem como participantes ativos em cuidar de pessoas com feridas e usar seu conhecimento sistemático para planejar o cuidado. No entanto, para aplicar o tratamento adequado, precisam de ferramentas eficientes para avaliar as condições de seus pacientes (SANTOS RC, et al., 2013).

Em estudo realizado por Dantas, et al. (2013), verificou-se que o diagnóstico da úlcera venosa é extremamente clínico, que inclui uma avaliação minuciosa da lesão e realização de diversos exames como hemograma completo, glicemia em jejum, albumina sérica, sinais vitais, cultura de exsudato, Índice Tornozelo Braquial (ITB), eco-doppler colorido, flebografia e pletismografia.

É de grande relevância a importância da atuação do enfermeiro ao considerar os aspectos socioeconômicos e clínicos ao avaliar o paciente portador de úlcera venosa, tendo em vista as repercussões que esse tratamento irá trazer na vida dos pacientes (MEDEIROS ABA, et al., 2016).

Dois itens se fazem extremamente importantes para o tratamento da úlcera venosa: o primeiro é a capacitação profissional que engloba vários fatores relacionados ao treinamento dos profissionais na realização do exame físico, da úlcera, do Índice Tornozelo Braço (TIB) e da terapia compressiva. O segundo se destaca o resumo clínico dos pacientes, resultados de exames, diagnóstico, tratamento, conduta e outras observações relevantes (DANTAS DV, et al., 2013).

Além disso, objetivando-se um tratamento ambulatorial adequado para os pacientes é de suma importância que as unidades de saúde tracem um plano de cuidados contínuo com o objetivo de dar continuidade ao tratamento e acessibilidade aos pacientes para as consultas de enfermagem. Nesse contexto, visando uma qualidade de vida mais satisfatória para os portadores de úlceras venosas, se faz necessário investir na meIhoria dos atendimentos clínicos, além de uma assistência de qualidade contínua e multiprofissional que visa atender todos os aspectos afetados pela lesão (OLIVEIRA FP, et al., 2016; DIAS TYAF, et al., 2013).

O tratamento ideal para úlcera venosa requer uma avaliação da lesão, exame físico completo, implementação de terapia que facilite o retorno venoso, uso de antibióticos, se necessário, visando um bom processo de cicatrização (REIS DB, et al., 2013).

Dentre os cuidados com a lesão Oliveira, et al. (2016), evidenciou intervenções de enfermagem segundo a Classificação das Intervenções de Enfermagem (NIC), quais sejam: o cuidado com as lesões, irrigação de lesões, administração de medicamentos, cuidados com a pele tratamentos tópicos, monitorização de sinais vitais, controle de hiperglicemia, controle da hemorragia, controle de medicamentos, supervisão, controle da nutrição, ensino sobre o cuidado dos pés e promoção do exercício.

Para desempenhar um bom atendimento além de intervenções sincronizadas, um protocolo com os seguintes itens viabiliza um atendimento de excelência: Avaliação do paciente e da lesão; Registro e documentação; Cuidado com a ferida e a pele perilesional; Indicação de cobertura; Uso de antibiótico e tratamento da dor; Tratamento cirúrgico da IVC; Tratamento medicamentoso; Melhorias do retorno venoso e prevenção de recidiva; Encaminhamento dos pacientes; Capacitação profissional e Referência e Contra- referência (DANTAS DV, et al., 2013).

Oliveira, et al. (2016), destaca ainda a assepsia da lesão com solução fisiológica a 0,9\%, técnica asséptica, uso de medicamentos específicos, registro da ferida como tamanho e aspecto são intervenções de enfermagem, assim como não se deve esquecer de avaliar a drenagem, a cor, o tamanho e odor. 
Visando uma melhoria do retorno venoso e a prevenção de recidiva, se faz necessário englobar diversas informações sobre a técnica e o profissional que aplica a terapia compressiva e estratégias clínicas e educativas para a prevenção. Um outro momento muito importante, é o encaminhamento desses pacientes para uma avaliação com angiologista, dermatologista e outros profissionais da equipe multidisciplinar (DANTAS DV, et al., 2013).

Entendendo-se que, não basta o acesso referenciado aos profissionais, faz-se necessário o registro nas fichas contendo resumo clínico, exames realizados, diagnóstico, conduta e observações pertinentes a cada caso (BAJAY HM, ARAÚJO IEM, 2006).

Vale ressaltar que o portador de úlcera deve estar atento a todas as orientações que são passadas, técnica correta para realização do curativo, a cobertura apropriada para a lesão, dieta que facilite o processo de cicatrização e uso de meias compressivas, evitando assim recidivas (REIS DB, et al., 2013).

O enfermeiro é responsável pelo diagnóstico dessas lesões, mas a falta de conhecimento no momento do diagnóstico acarreta várias consequências para o paciente. A educação continuada e protocolos instalados nas instituições ainda é a melhor forma para os profissionais obterem conhecimento acerca da patologia e orientar com discernimento e coerência os pacientes portadores dessas lesões.

\section{CONCLUSÃO}

O presente estudo possibilitou conhecer o perfil sociodemográfico do idoso portador de úlcera venosa e identificar o atendimento prestado pelo enfermeiro aos mesmos. Percebeu-se que a maioria deles apresenta um baixo nível de escolaridade, o que compromete a capacidade de entendimento acerca do autocuidado, 0 que pactua negativamente no tratamento.

O enfermeiro é responsável pelo diagnóstico dessas lesões, acompanhamento e orientação de todo o tratamento prestado a estes. Entretanto, ao apresentar déficit de conhecimento acerca da problemática, ocorre a influência negativa no processo de cicatrização das lesões, cooperando para a agravamento do sofrimento biopsicossocial do paciente e seus familiares. Logo, percebe-se que a atividade continuada e a implantação de protocolos direcionado ao atendimento às pessoas com úlceras venosas é a melhor solução para bom atendimento evitando assim recidivas.

\section{REFERÊNCIAS}

1. SANTANA RF, ALMEIDA KS, SAVOLDI NAM. Indicativos de Aplicabilidade das Orientações no Cotidiano de Cuidadores de Portadores de Alzheimer. Rev Esc Enferm USP 2009; 43(2):459-64.

2. MIRANDA GMD, MENDES ACG, SILVA ALA. O envelhecimento populacional brasileiro: desafios e consequências sociais atuais e futuras. Rev. Bras. Geriat. Geronto, 2016; 19(3): 507-519.

3. BRASIL (Ministério da Saúde). DATASUS. Indicadores de dados básicos - Brasil - 2008. Brasília: Ministério da Saúde; 2008.

4. ABBADE LPF, LASTORIA S. Abordagem de pacientes com úlcera da perna de etiologia venosa. An Bras Dermato 2006;81 (6): 509-22.

5. FERNANDES LF, PIMENTA FC, FERNANDES FF. Isolamento e perfil de suscetibilidade de bactérias de pé diabético e úlceras de estase venosa de pacientes admitidos no pronto-socorro do principal hospital universitário do estado de Goiás, Brasil. J Vasc Bras 2007;6(3):211-217.

6. SILVA FAA, FREITAS CHA, JORGE MSB, et al. Enfermagem em estomaterapia: cuidados clínicos ao portador de úlcera venosa. Rev Bras Enferm 2009; 62(6):889-93.

7. MALAGUTTI W, KAKIHARA TC. Curativos, Estomia e Dermatologia: Uma abordagem multiprofisisonal. 2 ed. São Paulo: Martinari,2011. Cap.6, p.73.

8. SANTOS RC, CAMACHO ACLF, VALENTE GSC, et al. Produção científica sobre cuidados de enfermagem aos pacientes adultos e idosos com úlceras venosas revisão integrativa. Rev enferm UFPE 2013; 7(esp): 4951-7.

9. OLIVEIRA FP, OLIVEIRA BGRB, SANTANA RF, et al. Classificações de intervenções e resultados de enfermagem em pacientes com feridas: mapeamento cruzado. Rev Gaúcha Enferm 2016; 37(2): e55033. 
10. BENEVIDES JP, COUTINHO JFV, SANTOS MCL, et al. Avaliação clínica de úlceras de perna em idosos. Rev Rene 2013; 13(2): 300-8.

11. EBERHARDT TD, LIMA SBS, LOPES LFD, et al. Perfil sociodemográfico e clínico de pacientes com úlceras venosas acompanhados em ambulatório: estudo transversal descritivo. Rev Enferm UFSM 2016; 6(4):539-547.

12. TORRES GV, BALDUINO LSC, COSTA IKF, et al. Comparação dos domínios da qualidade de vida de clientes com úlcera venosa. Rev Enferm UERJ 2014; 22(1): 57-64.

13. SALVETTI MG, COSTA IKF, DANTAS DV, et al. Prevalência da dor e fatores associados em pacientes com úlcera venosa. Rev Dor 2014; 15(1):17-20.

14. DIAS TYAF, COSTA IKF, SALVETTI MG, et al. Influência da assistência e características clínica na qualidade de vida de portadores de úlcera venosa. Acta Paul Enferm 2013; 26(6): 529-34.

15. SILVA MH, JESUS MCP, MERIGHI MAB, et al. O cotidiano do homem que convive com a úlcera venosa crônica: estudo fenomenológico. Rev Gaúcha Enferm 2013; 34(3): 95-101.

16. CARMO SS, CASTRO CD, RIOS VS, et al. Atualidade na assistência de enfermagem a portadores de úlcera venosa. Rev. Eletr. Enf. 2007; 9(2) 506-17.

17. MEDEIROS ABA, FRAZÃO CMFQ, FERNANDES MICD, et al. Associação dos fatores socioeconômicos e clínicos e o resultado integridade tissular em pacientes com úlceras. Rev Gaúcha Enferm 2016; 37(1): e54105.

18. RIZZATTI SJS, BUDÓ MLD, SILVA DC, et al. Cuidados em saúde de pessoas com úlcera venosa em assistência ambulatorial. Rev Enferm UFPI 2016; 5(1):17-22.

19. REIS DB, PERES GA, ZUFFli FB, et al. Cuidados às pessoas com úlcera venosa: Percepção dos enfermeiros da estratégia de saúde de família. Rev Min Enferm 2013; 17(1): 101-106.

20. DANTAS DV, DANTAS RAN, COSTA IKF, et al. Protocolo de assistência a pessoas com úlceras venosas: validação de conteúdo. Rev Rene 2013; 14(3): 588-99.

21. DANTAS DV, DANTAS RAN, ARAÚJO RO, et al. Proposta de protocolo para assistência as pessoas com úlceras venosas. Rev Enferm UFSM 2013; 3(Esp):618-626.

22. BAJAY HM, ARAÚJO IEM. Validação e confiabilidade de um instrumento de avaliação de feridas. Acta Paul Enferm. 2006: 19(3); 290-5. 